\title{
Follow-up after lung cancer resection: is intensified also justified?
}

\author{
Paul E. Van Schil
}

Affiliation: Dept of Thoracic and Vascular Surgery, Antwerp University Hospital, Edegem, Belgium.

Correspondence: P.E Van Schil, Dept of Thoracic and Vascular Surgery, Antwerp University Hospital, Wilrijkstraat 10, B-2650 Edegem (Antwerp), Belgium. E-mail: paul.van.schilauza.be

○ @ERSpublications

Intensified follow-up after lung cancer resection may be justified to detect recurrences at an early stage http://ow.ly/nKvlz

In patients who undergo complete resection of a nonsmall cell lung cancer (NSCLC) post-operative followup to detect locoregional and distant recurrences is indicated in order to find potentially treatable lesions at an early stage. There is also an increased risk of developing a second primary lung cancer, unrelated to the previous tumour. Thus, secondary screening may also be worthwhile in this patient population.

The questions relating to which examinations to perform, their optimal frequency, and precise duration of follow-up have not been clearly determined yet. Clinical examination and chest radiography are the basic, classical examinations performed. No doubt, chest computed tomography (CT) providing detailed lung and mediastinal images is more accurate than chest radiography. For primary screening, results of chest CT are quite impressive, as has been shown by the National Lung Screening Trial [1]. More than 50000 persons at high risk for developing lung cancer were randomised between screening with postero-anterior chest radiography and low-dose CT. In the CT arm a relative reduction of $20 \%$ in mortality from lung cancer was obtained. Also, the rate of death from any cause was reduced in the low-dose CT arm [1]. However, the jury is still out for secondary screening and follow-up after lung cancer resection. In a recent review, SCHMIDT-HANSEN et al. [2] concluded that no specific guidelines can be put forward on the most effective follow-up strategy and that there is an urgent need for further research on this subject. As follow-up represents a common clinical problem in daily practice, high-quality and validated data are needed.

In a present retrospective study in this issue of the European Respiratory Journal, GOURCEROL et al. [3] applied an extensive follow-up scheme in patients who underwent complete resection of a NSCLC. Every 3 months physical examination and chest CT were performed in 162 patients. Additional examinations included chest CT, bronchoscopy, abdominal ultrasound, CT scan of the brain and bone scanning every 6 months for 3 years, followed by yearly examinations for a period of 2 years. Recurrence occurred in 85 $(52.5 \%)$ patients, of which 41 were symptomatic. Disease-free survival was not different between symptomatic and asymptomatic patients. Recurrence was detected by physical examination or chest radiography in $55.3 \%$ of patients. Median overall survival from time of recurrence was 15.5 months in asymptomatic patients compared to only 7.2 months in symptomatic patients $(p=0.001)$. In multivariate analysis the procedures to diagnose recurrence and the absence of symptoms at the time of recurrence were significant factors. The authors included a detailed cost analysis and calculated a cost of $€ 22397$ per life year gained. They concluded that extensive follow-up may improve outcome of patients with resected NSCLC through detection of asymptomatic recurrences [3]. These results are quite interesting and may provoke a paradigm shift. However, this study has several limitations including its retrospective nature, the absence of a control group, long study duration, use of previous TNM (tumour, node, metastasis) classification and the fact that performance status at time of recurrence could not be precisely determined.

Received: May 182013 | Accepted: May 202013

Conflict of interest: None declared.

Copyright @ERS 2013 
Moreover, brain CT was only performed as planned in $24 \%$ of cases. Positron-emission tomography scanning, which may detect additional recurrences or second primary cancers, was not used during the study period.

So, validation of these results by prospective studies is certainly required and a large randomised study is currently being conducted in France. In the Intergroupe Francophone de Cancerologie Thoracique (IFCT)-0302 trial two follow-up schedules are being compared in patients who underwent complete resection of a NSCLC. One arm is undergoing simple, classical follow-up consisting of clinical examination and chest radiography, and the second arm is undergoing intensified follow-up with chest CT and bronchoscopy. The target population has currently been reached with 1865 included patients (V. Westeel, Centre Hospitalier Régional Universitaire de Besançon, Université de Franche-Comté, France; personal communication). Definite results of this study will not be available for several years but they are eagerly awaited in order to make more definite recommendations on the follow-up of lung cancer patients who undergo complete resection. Hopefully, a precise answer will be provided on whether intensified follow-up is able to detect asymptomatic recurrences and second primaries at an early stage with a better outcome in patients who can be treated with curative intent. In the meantime a scheme consisting of a follow-up visit every 3-6 months is recommended during the initial 2-3 years, and annually thereafter. Regarding follow-up examinations, history, physical examination, chest radiography and annual chest CT are appropriate tools. Smoking cessation is also essential after lung cancer resection as a better outcome is observed in patients who quit smoking at any time-point after the diagnosis of lung cancer [4]. So, most probably, an intensified follow-up schedule together with smoking counselling seems justified but level A evidence is hitherto lacking.

\section{References}

1 Aberle DR, Adams AM, Berg CD, et al. Reduced lung-cancer mortality with low-dose computed tomographic screening. N Engl J Med 2011; 365: 395-409.

2 Schmidt-Hansen M, Baldwin DR, Hasler E. What is the most effective follow-up model for lung cancer patients? A systematic review. J Thorac Oncol 2012; 7: 821-824.

3 Gourcerol D, Scherpereel A, Debeugny S, et al. Relevance of an extensive follow-up after surgery for nonsmall cell lung cancer. Eur Respir J 2013; 42: 1357-1364.

4 Balduyck B, Sardari Nia P, Cogen A, et al. The effect of smoking cessation on quality of life after lung cancer surgery. Eur J Cardiothorac Surg 2011; 40: 1432-1437. 